\title{
Formal degree and Clebsch-Gordan coefficient
}

\author{
By
}

Nobuhiko TATSUUMA

(Communicated by Prof. H. Yoshizawa, March 31, 1977)

1. Let $G$ be a unimodular locally compact group, and $\omega=\left\{\mathfrak{S}_{\mathfrak{g}}\right\}$ be a unitary representation of $G$. Here $\mathfrak{S}$ is the space of representation $\omega$ and $U_{g}^{\prime}$ 's are its representation operators.

We call $\omega L^{2}$-representation if and only if $\omega$ is irreducible and there exists a non-zero vector $v$ in $\mathfrak{S}$ such that $\left\langle U_{g} v, v\right\rangle$ is a square integrable function of $g$ in $G$ with respect to the right Haar measure $d g$ on $G$.

For an $L^{2}$-representation $\omega$, the following properties are known (cf. [1]).

1) For any vectors $u$, $w$ in $\mathfrak{g},\left\langle U_{g} u, w\right\rangle$ is square integrable.

2) For a fixed non-zero vector $v$ in $\mathfrak{S}$, the map

$$
\mathfrak{S} \ni u \longrightarrow\left\langle U_{\mathrm{g}} u, v\right\rangle \in L^{2}(G)
$$

is an intertwining operator from $\omega$ to the right regular representation $\Re=\left\{L^{2}(G), R_{8}\right\}$ of $G$.

3) For any representation $\tau=\left\{\Omega, V_{g}\right\}$ which is disjoint to $\omega$, and any vectors $u, v$ in $\mathfrak{C}$, any vectors $x, y$ in $\Re$ for which $\left\langle V_{g} x, y\right\rangle$ is square integrable,

$$
\int_{G}\left\langle U_{g} u, v><\overline{V_{g}} \bar{x}, \bar{y}\right\rangle d g=0 .
$$

4) There exists a positive number $d(\omega)$, depending only on $\omega$, such that

$$
\int_{G}\left\langle U_{g} u, v>\left\langle\overline{U_{g} w, z}\right\rangle d g=d(\omega)^{-1}\langle u, w\rangle\langle z, v\rangle\right.
$$

for any $u, v, w, z$ in $\mathfrak{Q}$. 
We call the number $d(\omega)$, the formal degree of $\omega$.

On the other hand, consider two unitary representations $\omega=\left\{\mathfrak{S}, U_{\mathfrak{g}}\right\}$, $\tau=\left\{\Omega, V_{g}\right\}$ and an irreducible one $\sigma=\left\{\mathcal{Q}, W_{g}\right\}$. Take normalized vectors $u, v, w$ in their representation spaces $\mathfrak{S}, \mathfrak{R}, \mathfrak{L}$ respectively.

Assume that $\omega \otimes \tau$ contains $\sigma$ as a discrete component. Denote $\tilde{L}$ the maximal subspace of $\mathfrak{S} \otimes \Re$ on which the restriction of $\omega \otimes \tau$ operates as a multiple of $\sigma$. It is evident that $\tilde{\mathfrak{L}}$ is uniquely determined invariant subspace, and the space of vectors

$\mathfrak{S}(w)=\{A w ; A$ is any intertwining operator from $\sigma$ to $\omega \otimes \tau\}$ is a closed subspace of $\tilde{\mathfrak{L}}$. Put $P_{\mathfrak{z}}$ and $P_{w}$ the projection of $\mathfrak{S} \otimes \mathfrak{R}$ to $\tilde{\mathfrak{L}}$ and $\mathfrak{S}(w)$ respectively.

We call the following non-negative real number the Clebsch-Gordan coefficient of $u \otimes v$ with respect to $w$ :

$$
\alpha(u, v ; w)=\left\|P_{w}(u \otimes v)\right\| .
$$

The purpose of this paper is to show a close relation between formal degrees and Clebsch-Gordan coefficients, and using this relation, to calculate the formal degree of the discrete series of $S L(2, R)$.

Hereafter we denote the conjugate representation of $\omega$ in the sense of G. W. Mackey by $\omega^{*}$, and the image of $v$ in $\mathfrak{g}$ by the natural conjugation map into the representation space $\mathfrak{S}^{*}$ of $\omega^{*}$ by $v^{*}$. For instance, $\left\langle U_{g}^{*} v^{*}, u^{*}\right\rangle=\left\langle\overline{U_{g} v, u}\right\rangle$. Obviously, if $\omega$ is an $L^{2}$-representation, $\omega^{*}$ is too and $d(w)=d\left(w^{*}\right)$ (cf. [2]).

2. Lemma 1. For any normalized vectors $u$ in $\mathfrak{E}, v$ in $\mathfrak{R}$, w in $\mathfrak{L}$,

$$
<\left(U_{g} \otimes V_{g}\right) P_{w}(u \otimes v), P_{w}(u \otimes v)>=\alpha(u, v ; w)^{2}\left\langle W_{g} w, w\right\rangle .
$$

Proof. From the definition of $\mathfrak{S}(w)$, there exists an intertwining isometric operator $A$ from $\mathfrak{L}$ into $\mathfrak{S} \otimes \mathbb{R}$ such that

$$
P_{w}(u \otimes v)=\alpha(u, v ; w) A w .
$$

This leads us to the above equality directly.

Lemma 2. The component of $\omega \otimes \tau$ restricted to the space $(\mathfrak{S} \otimes \mathbb{R}) \ominus \tilde{\mathbb{Q}}$ is disjoint to $\sigma$.

Proof. Obvious from the assumption of maximality on $\tilde{\mathfrak{L}}$.

3. Main Theorem. Let $\omega=\left\{\mathfrak{Q}, U_{g}\right\}$ be a unitary representation of $G$, and $\tau=\left\{\Re, V_{s}\right\}, \sigma=\left\{\Omega, W_{g}\right\}$ be two $L^{2}$-representations of $G$.

Assume $\omega \otimes \tau$ contains $\sigma^{*}$ as a discrete component. Then for any normalized vectors $u_{j}$ in $\mathfrak{S}, v_{j}$ in $\Re, w_{j}$ in $\mathfrak{Q}(j=1,2)$,

1) $\omega \otimes \sigma$ contains $\tau^{*}$ as a discrete component, 
2) $\alpha\left(u_{1}, v_{1} ; w_{1}^{*}\right)=\alpha\left(u_{1}^{*}, v_{1}^{*} ; w_{1}\right)$,

3) $d\left(\tau^{*}\right) \alpha\left(u_{1}, v_{1} ; w_{1}^{*}\right)^{2}=d\left(\sigma^{*}\right) \alpha\left(u_{1}, w_{1} ; v_{1}^{*}\right)^{2}$,

4) $\alpha\left(u_{1}, v_{1} ; w_{1}^{*}\right) \alpha\left(u_{2}, w_{2} ; v_{2}^{*}\right)=\alpha\left(u_{1}, w_{1} ; v_{1}^{*}\right) \alpha\left(u_{2}, v_{2} ; w_{2}^{*}\right)$.

Proof. At first the equality 2) is a direct conclusion of the definitions of $\alpha$ and the conjugation map.

For normalized vectors $u$ in $\mathfrak{E}, v$ in $\Omega$, $w$ in $\mathfrak{L}$, put

$$
\begin{aligned}
I_{0} & =\int_{G}\left\langle U_{g} u, u>\left\langle V_{g} v, v\right\rangle\left\langle W_{g} w, w\right\rangle d g\right. \\
& =\int_{G}\left\langle\left(U_{g} \otimes V_{g}\right)(u \otimes v), u \otimes v\right\rangle\left\langle\overline{W_{g}^{*} w^{*}, w^{*}}\right\rangle d g .
\end{aligned}
$$

Now we put $z_{1}=P_{w *}(u \otimes v), z_{2}=\left(P_{\mathfrak{1} *}-P_{w} *\right)(u \otimes v)$ and $z_{3}=\left(I-P_{2 *}\right)$ $(u \otimes v)$, then $z_{j}^{\prime} s$ are mutually orthogonal and $u \otimes v=z_{1}+z_{2}+z_{3}$. By lemma 1 ,

$$
<\left(U_{g} \otimes V_{g}\right) z_{1}, z_{1}>=\alpha\left(u, v ; w^{*}\right)^{2}<W_{g}^{*} w^{*}, w^{*}>\text {. }
$$

The vectors $z_{1}$ and $z_{2}$ are of the $\sigma^{*}$-component in $\omega \otimes \tau$, therefore the functions $\left\langle\left(U_{g} \otimes V_{g}\right) z_{j}, z_{k}\right\rangle(j, k=1,2)$ are square integrable. And since $\tilde{\mathfrak{L}}^{*}$ is an invariant subspace,

$$
\left\langle\left(U_{g} \otimes V_{g}\right) z_{3}, z_{j}\right\rangle=\left\langle\left(U_{g} \otimes V_{g}\right) z_{j}, z_{3}>=0 \quad(j=1,2) .\right.
$$

Combining these results, we obtain the following,

$$
\begin{aligned}
\left\langle U_{g} u, u>\left\langle V_{g} v, v>\right.\right. & =<\left(U_{g} \otimes V_{g}\right)(u \otimes v), u \otimes v>= \\
& =\sum_{j, k=1,2}\left\langle\left(U_{g} \otimes V_{g}\right) z_{j}, z_{k}>+<\left(U_{g} \otimes V_{g}\right) z_{3}, z_{3}>.\right.
\end{aligned}
$$

Because the left hand side and the first sum part of the right hand side are square integrable, the last term is too.

Thus by the orthogonality relations 3) and 4) in 1.,

$$
\begin{aligned}
& \int_{G}<\left(U_{g} \otimes V_{g}\right) z_{j}, z_{k}><\overline{W_{g}^{*} w^{*}, w^{*}}>d g=0 \quad(\text { if } j \text { or } k=2) . \\
& \int_{G}<\left(U_{g} \otimes V_{g}\right) z_{3}, z_{3}><\overline{W_{g}^{*} w^{*}, w^{*}}>d g=0 .
\end{aligned}
$$

Consequently we get

$$
I_{0}=\int_{G}\left\langle\left(U_{g} \otimes V_{g}\right) z_{1}, z_{1}><\overline{W_{g}^{*} w^{*}, w^{*}}>d g=\alpha\left(u, v ; w^{*}\right)^{2} d\left(\sigma^{*}\right)^{-1}\right.
$$

Changing the roll of $(\sigma, w)$ to $(\tau, v)$, we get analogously $I_{0}=\alpha\left(u, w ; v^{*}\right)^{2} d\left(\tau^{*}\right)^{-1}$.

The rest of Main theorem are deduced from this equality immedia- 
tely.

4. Now we shall consider the case of $G=S L(2, R)$. Its $L^{2}$-representations $D_{n}^{+}, D_{n}^{-}(n=1,3 / 2,2,5 / 2, \ldots)$ are so-called of the discrete series. In the space of the representation $D_{n}^{+}$(resp. $D_{n}^{-}$), there exists a complete orthonormal system $\left\{u_{n}^{j} ; j=n, n+1, \ldots\right.$ (resp. $u_{-n}^{j} ; j=-n$, $-n-1, \ldots)\}$ consisting of $K$-finite vectors.

For such vectors, the step-up (-down) operators are given by

$$
\begin{aligned}
& F^{+}\left(D_{n}^{ \pm}\right) u_{ \pm n}^{j}=\sqrt{(j+n)(j-n+1)} u_{ \pm n}^{j+1}, \\
& F^{-}\left(D_{n}^{ \pm}\right) u_{ \pm n}^{j}=-\sqrt{(j-n)(j+n-1)} u_{ \pm n}^{j-1} .
\end{aligned}
$$

The vector $u_{n}^{n}$ (resp. $u_{-n}^{-n}$ ) in the space of $D_{n}^{+}$(resp. $D_{n}^{-}$) is characterized as the normalized vector of weight $n$ (resp. $-n$ ) such that $F^{-}\left(D_{n}^{+}\right) u_{n}^{n}=0$ (resp. $F^{+}\left(D_{n}^{-}\right) u_{-n}^{-n}=0$ ) up to constant factor (cf. [3]).

In the space of $D_{1 / 2}^{+} \otimes D_{p}^{+}$, the vector $u_{1 / 2}^{1 / 2} \otimes u_{p}^{p}$ is the only normalized vector of weight $p+(1 / 2)$ and $F^{-}\left(D_{1 / 2}^{+} \otimes D_{p}^{+}\right)\left(u_{1 / 2}^{1 / 2} \otimes u_{p}^{p}\right)=0$. This means that $D_{1 / 2}^{+} \otimes D_{p}^{+}$contains $D_{p+(1 / 2)}^{+}$with multiplicity one and $u_{1 / 2}^{1 / 2} \otimes u_{p}^{p}$ just corresponds to the vector $u_{p+1}^{p+(1 / 2)}$. That is,

$$
\alpha\left(u_{1 / 2}^{1 / 2}, u_{p}^{p} ; u_{p+(1 / 2)}^{p+(1 / 2)}\right)=\alpha\left(u_{1 / 2}^{1 / 2}, u_{p}^{p} ;\left(u_{-p-(1 / 2)}^{-p-(1 / 2)}\right)^{*}\right)=1 \text {. }
$$

While in the space of $D_{1 / 2}^{+} \otimes D_{-p-C_{1 / 2)}}^{-}=D_{1 / 2}^{+} \otimes\left(D_{p+(1 / 2)}^{+}\right)^{*}$, vectors $v$ of weight $-p$ are given by

$$
v=\sum_{l \geq 0} a_{l}\left(u_{1 / 2}^{l+(1 / 2)} \otimes u_{-p-(1 / 2)}^{-p-l-(1 / 2)}\right) .
$$

Thus

$$
\begin{aligned}
F^{+} v & =\sum_{l \geq 0} a_{l}\left((l+1) u^{l+(3 / 2)} \underset{1 / 2}{1 / 2} \otimes u_{-p-(1 / 2)}^{-p-l-(1 / 2)}+\right. \\
& \left.+\sqrt{l(l+2 p)} u^{l+1 / 2} \otimes u_{-p-(1 / 2)}^{-p-l+(1 / 2)}\right) \\
& =\sum_{l \geq 0}\left(a_{l}(l+1)+a_{l+1} \sqrt{(l+1)(l+2 p+1))}\left(u_{\substack{l+(3 / 2) \\
1 / 2}} \otimes u_{-p-(1 / 2)}^{-p-l(-1 / 2)}\right) .\right.
\end{aligned}
$$

The equality $F^{+} v=0$ gives $a_{l+1}=-a_{l} \sqrt{(l+1) /(l+2 p+1)}$. Therefore $a_{l}=$ $(-1)^{l} \sqrt{l !(2 p) ! /(l+2 p) !} a_{0}(l \geqq 0)$. By the additional condition $\|v\|=1$, we get

$$
a_{0}=\alpha\left(u_{1 / 2}^{1 / 2}, u_{-p-(1 / 2)}^{-p-(1 / 2)} ; u_{-p}^{-p}\right)=((2 p-1) / 2 p)^{1 / 2} .
$$

Substitute this to the formula 3) of the Main theorem, then we obtain

$$
\begin{aligned}
& d\left(D_{p}^{-}\right) / d\left(D_{p+(1 / 2)}^{+}\right)=d\left(D_{p}^{+}\right) / d\left(D_{p+(1 / 2)}^{+}\right)= \\
& =\alpha\left(u_{1 / 2}^{1 / 2}, u_{-p-(1 / 2)}^{-p-(1 / 2)} ; u_{-p}^{-p}\right)^{2} / \alpha\left(u_{1 / 2}^{1 / 2}, u_{p}^{p} ; u_{p+(1 / 2)}^{p+(1 / 2)}\right)^{2}=(2 p-1) / 2 p \\
& (p=1,3 / 2,2,5 / 2, \ldots) .
\end{aligned}
$$


Summarizing these results, if we put $b_{0}=d\left(D_{1}^{+}\right)$,

$$
d\left(D_{p}^{+}\right)=d\left(D_{p}^{-}\right)=(2 p-1) b_{0}(p=1,3 / 2,2,5 / 2, \ldots) .
$$

The determination of $b_{0}$ depends of the normalization of the Haar measure on $G$. For instance, for the normalization as

$$
\begin{aligned}
& \int_{G}\left|\left\langle U_{\boldsymbol{g}} u_{1}^{1}, u_{1}^{1}\right\rangle\right|^{2} d g\left(=d\left(D_{1}^{+}\right)\right)=1, \text { we get } \\
& d\left(D_{p}^{+}\right)= d\left(D_{p}^{-}\right)=(2 p-1) . \\
& \text { DEPARTMENT OF MATHEMATICS, } \\
& \text { KYOTO UNIVERSITY }
\end{aligned}
$$

\section{References}

[1] G. Warner, Harmonic Analysis on Semisimple Lie Groups I, Springer-Verlag Berlin, 1972.

[2] G. W. Mackey, Induced representations of locally compact groups I, Ann. of Math., 55 (1952), pp. 101-139.

[ 3 ] N. Tatsuuma, A duality theorem for the real unimodular group of second order, J. Math. Soc. Japan, 17 (1965) pp. 313-332. 